\title{
Adaptive Rest Condition Potentials: Second Order Edge-Preserving Regularization
}

\author{
Mariano Rivera and Jose L. Marroquin \\ Centro de Investigacion en Matematicas A.C. \\ Apdo. Postal 402, Guanajuato, Gto., 36000, Mexico \\ $\{$ mrivera, jlm\}@cimat.mx \\ http://www. cimat.mx/ ${ }^{\sim m r i v e r a}$
}

\begin{abstract}
The propose of this paper is to introduce a new regularization formulation for inverse problems in computer vision and image processing that allows one to reconstruct second order piecewise smooth images, that is, images consisting of an assembly of regions with almost constant value, almost constant slope or almost constant curvature. This formulation is based on the idea of using potential functions that correspond to springs or thin plates with an adaptive rest condition. Efficient algorithms for computing the solution, and examples illustrating the performance of this scheme, compared with other known regularization schemes are presented as well.
\end{abstract}

Keywords. Edge-preserving regularization, image restoration, segmentation, anisotropic diffusion.

\section{Introduction}

In recent years, several methods for Edge-Preserving Regularization (EPR) for inverse problems in computer vision and image processing, have been published. These EPR methods are based on potentials that grow at a slower rate than quadratic ones. These methods have demonstrated their performance in detecting outliers in the data and reconstructing piecewise smooth images. The definition of piecewise smooth, however, has in most cases meant "almost piecewise constant", which means that the image can be represented as an assembly of regions such that inside them the gradient is close to zero. In the regularization framework, given the observed image $g$, the regularized solution $f$ is computed as the minimizer of an energy functional $U$. Given a good initial guess for $f$, efficient algorithms for computing a local minimum have been reported in the literature. In spite of the success of robust regularization methods, there are still open important problems; in particular, the definition of piecewise smooth images has not been extended successfully to include regions with almost constant slope (second order smoothness). As a result, regions with constant slope are reconstructed with a "staircase" effect.

The purpose of this paper is to introduce a new formulation for energy potentials that allows one to reconstruct images with second order piecewise smoothness. In addition, efficient algorithms for computing the solution are presented. 
The organization of the paper is as follows: section 2 presents a review of the EPR techniques based on robust potentials. In order to clarify the behavior of first order robust regularization an analogy with a Weak Spring System (WSS) is used. We show that this model has limitations for representing potentials for high order EPR.

In the third section, the new potentials for second order EPR are introduced. For first order potentials, the corresponding analogous model is a spring system with adaptive rest condition (ARC); we show that for this case, there is an equivalence between ARC and WSS potentials; however, ARC potentials can naturally be extended to high order EPR potentials, which do not have a direct representation in the WSS model.

Minimization algorithms are presented in section four and in section five, the performance of the proposed ARC potentials is demonstrated by experiments in both synthetic and real data. Finally, our conclusions are given in section six.

\section{Robust Regularization}

\subsection{Statement of the Problem}

The problem of reconstructing an image $\widehat{f}$ from noisy and degraded observations $g$ given the following model of the observations:

$$
g=F(\widehat{f})+\eta
$$

where $\eta$ is additive noise and $F$ is (in general) a non-linear operator that is assumed to be known, is an ill posed problem. Therefore, regularization of the problem is necessary. This means that, prior information or assumptions about the structure of $\widehat{f}$ need to be introduced in the reconstruction process. The regularized solution $f^{*}$ is computed by minimizing an energy functional $U$ :

$$
f^{*}=\arg \min _{f} U(f)
$$

where $U$ is of the form:

$$
U(f)=D(f, g)+\lambda R(f)
$$

The first term in (2) establishes that the reconstructed $f$ should be consistent with the data $g$ and the second term imposes a penalty for violating the prior assumptions of piecewise smoothness. The relative contribution of each term to the global energy is controlled by the positive parameter $\lambda$.

\subsection{The Homogeneous Spring System}

In the framework of Bayesian regularization, the data term in (2) is chosen as the negative log-likelihood and the prior constraints are incorporated in the form of a prior MRF model for $f$ [1], so that the regularization term $R$ in (2) takes the form of a sum, over the cliques of a given neighborhood system, of a set of 
"potential functions" supported on those cliques. One may take for instance as the neighborhood $N$ of a pixel $r$ its 8 closest neighbors:

$$
N_{r}=\{s:|r-s|<2\}
$$

and cliques of size $2\langle r, s\rangle$ that correspond to horizontal, vertical and diagonal pixel pairs, where $r=(x, y)$ represents a site in the pixel lattice $L$. A quadratic regularized potential is obtained by assuming that $\eta$ corresponds to Gaussian noise and choosing quadratic potentials over the first neighbor pairs:

$$
U_{H}(f)=\sum_{r}\left\{\left|F(f)_{r}-g_{r}\right|^{2}+\frac{\lambda}{2} \sum_{s \in N_{r}} d_{r s}\left|f_{r}-f_{s}\right|^{2}\right\}
$$

with $d_{r s}=|r-s|^{-1}$. Functional (3) corresponds to the internal energy of the physical model of a Homogeneous Spring System (HSS). The HSS model is equivalent to a system of particles located at the sites of the pixel lattice, so that the vertical position of each particle is represented by the gray level of each pixel. Eq. (3) corresponds to the energy of the complete system where (when $F$ is the identity) each particle $f_{r}$ is connected by means of springs with the observation $g_{r}$ and with its neighboring particles. The cost functional (3) does not preserve edges and will produce an over-smoothing of the real edges of the image.

\subsection{The Weak Spring System: Robust Regularization}

To alleviate that problem, there have been proposed potential functions for the regularization term that allow edge preservation, based on the idea of a breakable spring, that is, if the potential energy of a spring exceeds a given threshold $\theta$, then the spring must be broken [2] or weakened [3] [4] 5] 6]. To achieve this behavior, an auxiliary variable $\omega$ than acts as edge (outlier) detector is introduced; then the potential takes the form:

$$
\rho\left(f_{r}-f_{s}, \omega_{r s}\right)=\omega_{r s}\left(f_{r}-f_{s}\right)^{2}+\Psi\left(\omega_{r s}\right)
$$

where $\omega_{r s}$ is associated to each pixel pair $(r, s)$, and $\Psi$ is a potential function that controls an over-detection of edges. In the case of the breakable spring model [2], $\omega_{r s}$ only takes the values $\{0,1\}$; on other hand, in the case of the WSS model, $\omega_{r s} \in[0,1]$, and is set close to 1 for $\left(f_{r}-f_{s}\right)^{2}<\theta$ (where $\theta$ is a given threshold) and less that one otherwise. Black and Rangarajan [4] have shown that the potentials of the weak spring model correspond to the cost function for robust M-estimators. These potentials are, in general, non-convex and grow at a slower rate than the quadratic ones. This method is capable of finding the significant missing data of a noisy image and performing an edge-preserving restoration. Furthermore, the explicit outlier detection formulation allows one to incorporate additional constraints about the structure of the edge reject field $\omega$ [4. For instance, one can penalize the "thickness" and the discontinuities on the edges, at the expense of an additional computational cost. 


\subsection{The Weak Thin Plate Model}

The thin plate model 13] is obtained when one uses as potentials, squares of finite difference approximations to second derivatives:

$$
\Delta^{2} f_{r}=f_{q}-2 f_{r}+f_{s} .
$$

The computation of $\Delta^{2} f_{r}$ involves cliques of size $3\langle q, r, s\rangle$ that correspond to horizontal, vertical and diagonals pixel triads (see figure 1).

$$
\begin{array}{ll}
\text { (a) } \stackrel{\mathrm{q} \cdot}{\mathrm{r}} \quad \text { (b) } \mathrm{q} \cdot \mathrm{r} \cdot \mathrm{s} \cdot \\
\mathrm{s} \cdot & \\
\text { (c) } & \mathrm{q} \cdot \mathrm{r}_{\mathrm{s}}^{\mathrm{r}} \quad \text { (d) } \mathrm{q} \cdot \mathrm{r} \cdot
\end{array}
$$

Fig. 1. Cliques with triads of pixels

One could use the weak potential

$$
\rho\left(\Delta^{2} f_{r}, \omega_{q r s}\right)=\left[\Delta^{2} f_{r}\right]^{2} \omega_{q r s}+\mu \Psi\left(\omega_{q r s}\right),
$$

as a second order edge-preserving potential; however, the results are not completely satisfactory, even for the reconstruction of piecewise constant images, where the first order model presents an excellent performance. The observed effect consists in the "ramping" or interpolation of first order discontinuities. In order to compute a second order solution, Geman and Reynolds [3] proposed to use the reconstruction computed with the first order model [using $\rho\left(f_{r}-f_{s}, \omega_{r s}\right)$ ] as the starting point for the second order model. This improves the results, but still presents some problems, because the outliers for the first order model (jumps) do not correspond to the outliers for the second order one (large curvatures). Thus, the weak second order model does not work properly in the edges defined by jumps in the gray level (see section 5 ).

\section{The Adaptive Rest Condition Potentials (ARC-Potentials)}

In this section, we introduce potentials for EPR that are based on the paradigm of the Adaptive Rest Condition (ARC). The system we are proposing is based on the idea of using quadratic potentials with a non-zero (adaptive) rest condition:

$$
\rho_{\text {arc }}\left(t, \phi_{t}\right)=\left|t-\phi_{t}\right|^{2}+\Phi\left(\phi_{t}\right),
$$

where $\phi_{t}$ acts as the signed rest condition of the potential $\rho_{\text {arc }}$. In Refs. 9] [10 a similar formulation is reported, in which $\phi$ are auxiliary variables introduced to 
minimize non-quadratic potentials by means of half-quadratic techniques - as in [3] 4] 5. Here, however, we introduce the ARC potentials specifically to produce a novel generalization to the second order case. In the first order case, in fact, ARC potentials are equivalent to existing EPR potentials, as we show in the next subsection.

\subsection{Spring System with Adaptive Rest Condition (SARC)}

Consider $t=f_{r}-f_{s}$, thus,

$$
\rho_{\text {arc }}\left(f_{r}-f_{s}, \phi_{r s}\right)=\left|f_{r}-f_{s}-\phi_{r s}\right|^{2}+\Phi\left(\phi_{r s}\right),
$$

Note that the desired behavior for the potential $\rho_{\text {arc }}$ corresponds to choosing $\phi_{r s}$ close to zero for those values of $f_{r}-f_{s}$ that we want to smooth out, and close to the value of $f_{r}-f_{s}$ for those values that are considered edges. $\Phi$ is a given function that penalizes rest conditions different from zero. Figure 2 (a) shows the plot for a typical $\phi$, and the corresponding ARC-potential is shown in panel 2-(b). As one can see the $\rho_{\text {arc }}$ potential has a quadratic region (for $|t|<0.5$ ) and outside this region an outlier rejection zone. In following we show how to choose the ARC and function $\Phi$ in order to have the desired effect.

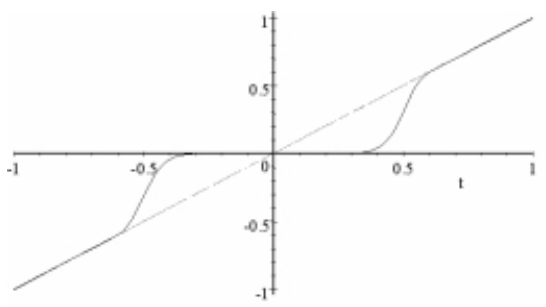

(a)

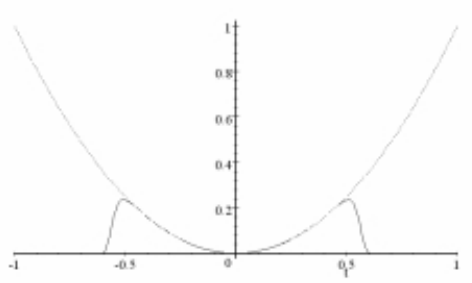

(b)

Fig. 2. (a) A typical rest condition $\phi$ (solid line) and the residual error $t=f_{r}-f_{s}$ (doted line). (b) ARC-Potential corresponding to $|t-\phi|^{2}$, with $\phi$ plotted in panel(a). Doted line: quadratic potential $t^{2}$

There are three possible strategies for choosing a spring system with ARC (SARC):

1. Explicit analogical line process (SARC-AL). In this case, one introduces auxiliary variables $l$, and chooses $\phi=l t$ and $\Phi(\phi)=\Psi(\phi / t)$ [see eq.(4)]. Where $\Psi$ is a convex function. In this case, the SARC-AL potential corresponds to a half-quadratic potential. For instance, if we use $\Phi(\phi)=\mu(\phi / t)^{2}$, where $\mu$ is a positive parameter, one obtains the Ambrosio-Tortorelli[11] potential:

$$
\rho_{\text {arc }}(t, l)=|t-l t|^{2}+\mu(l)^{2} .
$$

Note that in this case the optimal $l$ is always in the interval $[0,1]$. 
2. Explicit binary line process (SARC-BL). In this case $l$ is binary valued $(l \in$ $\{0,1\})$ and $\Phi(t)=\mu V(t)$ where $V$ is the Ising potential [1]:

$$
V(t)= \begin{cases}-1 & \text { if } t=0 \\ 1 & \text { otherwise }\end{cases}
$$

In this case, SARC-BL is equivalent to the breakable spring model 2].

3. Implicit line process (SARC-IL). In this case the ARC potential takes the form:

$$
\rho_{\text {arc }}(t)=|t-\phi(t)|^{2}
$$

where $\phi$ is a given function shaped as the one plotted in Figure 2. For instance, if one chooses $\phi(t)=t-\sqrt{\rho(t)}$, where $\rho$ is a robust potential, we have $\rho_{\text {arc }}(t)=\rho(t)$, so that SARC-IL is equivalent to the robust potential formulation.

Although SARC models are equivalent to existing EPR techniques, we can obtain an extra benefit by using a combination of explicit and implicit line detection [12]:

$$
\rho_{\text {arc }}(t, l)=|t-l \phi(t)|^{2}+\frac{1}{\mu}(l)^{2} .
$$

where $\mu$ is positive parameter and the product $l \phi(t)$ is the ARC. The meaning of the extra variable $l$ depends on the chosen function $\phi$. In order to illustrate this combined formulation, one can assume that $\phi(t)$ corresponds to the function plotted in Fig. 2. In this case, for a small value of the parameter $\mu$ and if $|t|<$ 0.5 , one has that $l$ is equal to zero (by effect of the penalization term). On the other hand, if $|t|>0.5$, then $l$ will be close to one. As a consequence, $l$ represents an edge detector controlled by the function $\phi$. This allows one to use $\phi$ to represent prior constraints about edge location; for example, in the edgepreserving regularization of optic flow, one may prevent edges from appearing in regions with small gray-level gradient by setting $\phi=0$ in those regions [12].

\subsection{Thin Plate System with Adaptive Rest Condition (PARC)}

The greatest advantage of the ARC formulation is that it can be used to produce a novel extension to the second order case, by defining thin plate potentials with adaptive rest condition. These PARC regularization potentials have the property of not just adapting their stiffness, but also changing their behavior to SARC potentials at the edges of almost constant regions. This represents a significant advantage over the half-quadratic plate model based on robust potentials $3 \mathbf{3} 45$, 7,8,9,10,13,14,15,16]

We have two cases for the PARC model: analogical line process (PARC-AL) and implicit line process (PARC-IL). In order to understand the PARC models, we first introduce the PARC-AL model.

We note that (5) can be written as:

$$
\Delta^{2} f_{r}=\Delta^{+} f_{r}-\Delta^{-} f_{r}
$$

where 


$$
\Delta^{+} f_{r}=f_{q}-f_{r} \quad \text { and } \quad \Delta^{-} f_{r}=f_{r}-f_{s}
$$

(see figure 1).

Then, PARC potentials can be written in the general form:

$$
\rho_{\text {parc }}\left(\Delta^{2} f_{r}\right)=\left|\Delta^{+} f_{r}-\Delta^{-} f_{r}-\phi_{r}\right|^{2}+\Phi\left(\phi_{r}\right),
$$

where the ARC $\phi$ should satisfy:

$$
\phi_{r} \approx\left\{\begin{array}{cll}
0 & \text { if }\left|\Delta^{+} f_{r}\right|<\theta, & \left|\Delta^{-} f_{r}\right|<\theta \\
\Delta^{-} f_{r} & \text { if }\left|\Delta^{+} f_{r}\right|<\theta, & \left|\Delta^{-} f_{r}\right|>\theta \\
\Delta^{+} f_{r} & \text { if }\left|\Delta^{+} f_{r}\right|>\theta, & \left|\Delta^{-} f_{r}\right|<\theta \\
\Delta^{2} f_{r} & \text { if }\left|\Delta^{+} f_{r}\right|>\theta, & \left|\Delta^{-} f_{r}\right|>\theta
\end{array},\right.
$$

where $\theta$ is a given threshold. To obtain the desired behavior, one may represent $\phi_{r}$ as: $\phi_{r} \equiv \phi_{r}^{+}+\phi_{r}^{-}$, where $\phi_{r}^{+}, \phi_{r}^{-}$depend on $\Delta^{+} f_{r}$ and $\Delta^{-} f_{r}$, respectively, and penalize $\phi_{r}^{+}$and $\phi_{r}^{-}$separately. Thus, (12) can be rewritten as:

$$
\rho_{\text {parc }}\left(\Delta^{2} f_{r}\right)=\left|\Delta^{+} f_{r}-\Delta^{-} f_{r}-\phi_{r}^{+}-\phi_{r}^{-}\right|^{2}+\Phi\left(\phi_{r}^{+}\right)+\Phi\left(\phi_{r}^{-}\right)
$$

One can now introduce "edge variables" $l_{q r}=\phi_{r}^{+} / \Delta^{+} f_{r}, l_{r s}=\phi_{r}^{-} / \Delta^{-} f_{r}$, so that (12) can be written as:

$$
\rho_{\text {parc }}(f, l)_{q r s}=\left|\Delta^{+} f_{r}\left(1-l_{q r}\right)-\Delta^{-} f_{r}\left(1-l_{r s}\right)\right|^{2}+\mu \Psi\left(l_{q s}\right)+\mu \Psi\left(l_{r s}\right)
$$

This potential is quadratic on $f$ for a given $l$ and satisfies the constraints (13) (see figure 3). $\Psi(\cdot)$ is a potential that penalizes an over-detection of edges, and must satisfy the constraints:

1. $\Psi(t) \geq 0 \forall t$ with $\Psi(0)=0$,

2. $\Psi(t)=\Psi(-t)$.

3. $\Psi^{\prime}(t) \equiv \partial \Psi(t(f)) / \partial f$ and $\Psi^{\prime \prime}(t) \equiv \partial^{2} \Psi(t(f)) / \partial f^{2}$ exist.

Note that there is no closed formula for $l_{q r}$ and $l_{r s}$. Unlike the SARC-AL model, in PARC-AL there is no guarantee that $l \in[0,1]$.

\subsection{PARC-IL Models}

The implicit line process PARC model (PARC-IL) is generated by using $\phi^{+}=$ $\phi\left(\Delta^{+} f_{r}\right)$ and $\phi^{-}=\phi\left(\Delta^{-} f_{r}\right)$, where we use

$$
\phi(t)=t(1-\psi(t))
$$

where $\psi$ is chosen in a way such that $t \psi(t)$ is close to $t$ for small values of $t$ and close to zero for large values of $t$ (we used $\psi(t)=\exp \left(-t^{2}\right)$ in our experiments of section IV). The edge penalization term takes in this case the form $\mu\left[\left|\Delta^{+} f_{r}\right|^{2}+\left|\Delta^{-} f_{r}\right|^{2}\right]$. Thus, the complete PARC-IL is given by

$$
\rho_{\text {parc }}(f)_{r}=\left|\Delta^{+} f_{r} \psi\left(\frac{\Delta^{+} f_{r}}{k}\right)-\Delta^{-} f_{r} \psi\left(\frac{\Delta^{-} f_{r}}{k}\right)\right|^{2}+\mu\left[\left|\Delta^{+} f_{r}\right|^{2}+\left|\Delta^{-} f_{r}\right|^{2}\right]
$$


where $k$ is a scale parameter and the parameter $\mu$ controls the granularity of the solution, since small regions surrounded by edges will be penalized by this term [13.

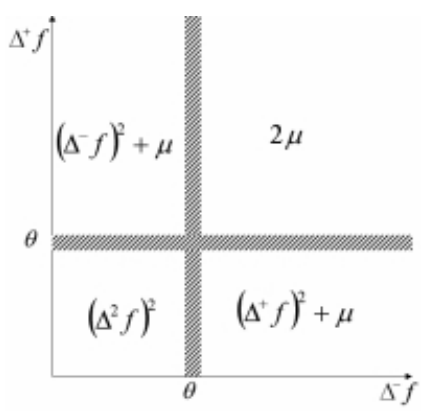

Fig. 3. Desired behavior of the PARC potential. For $\Delta^{+} f>\theta$ and $\Delta^{-} f>\theta(\theta$ is a given threshold) the potential is quadratic. $\rho_{\text {arc }}=\left|\Delta^{-} f\right|^{2}+\mu$ if $\Delta^{+} f>\theta$ and $\Delta^{-} f$ $<\theta$. $\rho_{\text {arc }}=\left|\Delta^{+} f\right|^{2}+\mu$ if $\Delta^{+} f<\theta$ and $\Delta^{-} f>\theta$. Finally, $\rho_{\text {arc }}=2 \mu$ if $\Delta^{+} f>\theta$ and $\Delta^{-} f>\theta$, where $\mu$ is a fixed cost.

\section{Minimization Algorithms}

\subsection{Half-Quadratic Coupled Minimization Algorithms for PARC-AL Models}

In this case, the function to minimize is:

$$
\begin{aligned}
U(f, l) & =\sum_{r}\left\{\left[F(f)_{r}-g_{r}\right]^{2}\right. \\
& +\lambda \sum_{q, s \in N_{r}}\left(\left[\Delta^{+} f_{r}\left(1-l_{q r}\right)-\Delta^{-} f_{r}\left(1-l_{r s}\right)\right]^{2}\right. \\
& \left.\left.+\mu\left[\Psi\left(l_{q r}\right)+\Psi\left(l_{r s}\right)\right]\right)\right\}
\end{aligned}
$$

where $F$ is a linear operator. The solution may be computed by a two-step iterative minimization algorithm:

1. Quadratic minimization step: Update the restoration $f^{t+1}$ (keeping $l$ fixed), so that:

$$
U\left(f^{t+1}, l^{t}\right) \leq U\left(f^{t}, l^{t}\right)
$$


2. Update the second order edge detectors $l$ (keeping $f$ fixed), so that:

$$
U\left(f^{t+1}, l^{t+1}\right) \leq U\left(f^{t+1}, l^{t}\right) .
$$

and set $t=t+1$.

For the special case $\Psi\left(l_{q r}\right)=l_{q r}^{2}$, (16) is quadratic for $f$ for a given $l$, and quadratic for $l$ for a given $f$. Thus, the alternated minimization of (16) is performed by solving a coupled linear systems using any general algorithm as GaussSeidel or linear Conjugate Gradient. We have found a very good performance by alternating a single Gauss-Seidel iteration of each system. The experiments of the figures 3 - (e) and 5 - (b) correspond to this simple case.

\subsection{Adaptive Non-linear Conjugate Gradient Algorithm (ANLCG) for PARC-IL Models}

The cost functional with implicit edge detection is given by

$$
\begin{aligned}
U(f) & =\sum_{r}\left\{\left[F(f)_{r}-g_{r}\right]^{2}\right. \\
& +\sum_{q, s \in N_{r}}\left(\left|\Delta^{+} f_{r} \psi\left(\frac{\Delta^{+} f_{r}}{k}\right)-\Delta^{-} f_{r} \psi\left(\frac{\Delta^{-} f_{r}}{k}\right)\right|^{2}\right. \\
& \left.\left.+\mu\left[\left|\Delta^{+} f_{r}\right|^{2}+\left|\Delta^{-} f_{r}\right|^{2}\right]\right)\right\}
\end{aligned}
$$

Note that $U$ is differentiable, but non-quadratic in $f$, so a non-linear optimization algorithm needs to be used to find a local minimum of $U$. We propose here a modification to the Non-Linear Conjugate Gradient Algorithm (ANLCG), in which the step size is adaptively varied. Additionally, in order to accelerate the convergence rate, the algorithm introduces inertia in the descent. The algorithm is:

\section{ANLCG}

Set $n=1, \beta_{0}=0, f_{0}$ equal to an initial guess, and $g_{0}=G\left(f_{0}\right)$

Repeat until $\left|g_{n}\right|<\varepsilon$ :

1. $s_{n}=-g_{n}+\beta_{n} s_{n-1}$

2. Compute the step $\alpha_{n}$ such that ensures energy reduction. i.e. $U\left(f_{n}+\alpha_{n} s_{n}\right)<$ $U\left(f_{n}\right)$ (see below)

3. $f_{n+1}=f_{n}+\alpha_{n} s_{n}$, $n=n+1$.

4. $g_{n}=\nabla U\left(f_{n}\right)$

5. $\beta_{n}=\max \left\{0, \frac{g_{n}^{T}\left(g_{n}-g_{n-1}\right)}{g_{n-1}^{T} g_{n-1}}\right\}$,

where $\varepsilon \in(0,1)$ is a small positive constant. For computing the step $\alpha_{n}$, we propose an algorithm that uses an adaptive local search. This algorithm is 
inspired in the one used in the Quasi-Gauss-Newton algorithm with an energy reduction constraint [17. ANLCG algorithm (Step 2) requires that $\alpha_{n}$ is accepted only if it guarantees a sufficient reduction in the energy $U(f)$, that is , if

$$
U\left(f_{n}+\alpha_{n} s_{n}\right) \leq(1-\varepsilon) U\left(f_{n}\right),
$$

to achieve this we do the following:

Computation of $\alpha_{n}$

Initially set $a=0.01, m=0$, and $\delta$ small enough (e.g. $\delta=10^{-4}$ ).

$2.1 \quad \alpha_{n}=a$

2.2 While $U\left(f_{n}+\alpha_{n} s_{n}\right)>\left(1-\alpha_{n} \delta\right) U\left(f_{n}\right)$

$2.3 \quad m=m+1$

$$
\alpha_{n}=\alpha_{n} / c_{1}, m=0
$$

$2.4 \quad$ if $m>c_{2}$

$$
a=c_{3} \alpha_{n}, m=0
$$

else

$$
a=\alpha_{n}
$$

Empirically, we have found that the values of parameters $c_{1}=2, c_{2}=5$ and $c_{3}=3$ and the initial step size $a$ work properly. Note that since $\alpha_{n}$ ensures that the energy decreases at every iteration, the convergence of algorithm ANLCG is automatically guaranteed.

\section{$5 \quad$ Experiments}

In this section experiments (in both synthetic and real data) that demonstrate the performance of the PARC potentials are shown.

\subsection{Comparison with the Half-Quadratic Second Order Model}

The first experiment is a comparison of the performance of the PARC models with respect to other EPR model: the proposed by Geman and Reynolds 3 . The synthetic test image is shown in panel (a) in figure 4, the noise corrupted image is shown in panel (b). The test image was designed so that includes the following kind of regions: piecewise constant (delimited by first order discontinuities: edges), piecewise constant slope (second order discontinuities) and smooth (non-planar) regions. Panel (d) shows the reconstructed (filtered) image computed with a first order EPR model (the WSS). One can appreciate that WSS models promotes constant piecewise restorations. Panel (d) shows the reconstruction computed with (in our knowledge) the only second order model reported (see ref. [3]). This corresponds to the weak thin plate model (WTP). The used potential function corresponds to the one reported in [7. This potential has been extensively used and has demonstrated its superior performance with respect to other first order EPR potentials (see [4] [5]). As one can see, in spite of the fact that the regions with constant and smooth changes in the slope are reconstructed with an acceptable quality, the gray level steps are over-smoothed. This 
(a)

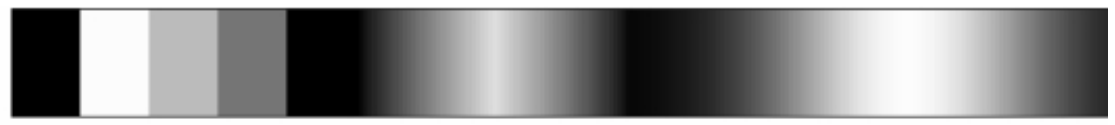

(b)

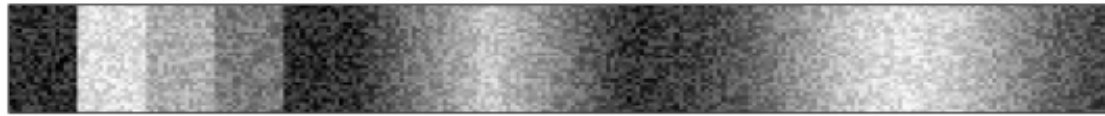

(c)

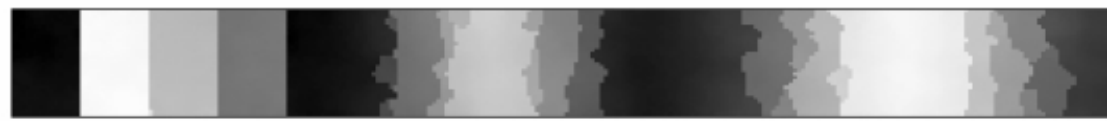

(d)

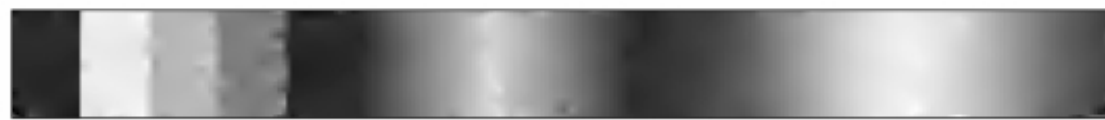

(e)

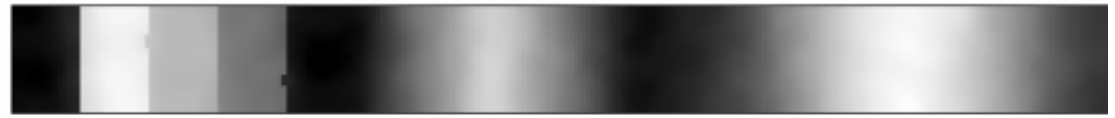

(f)

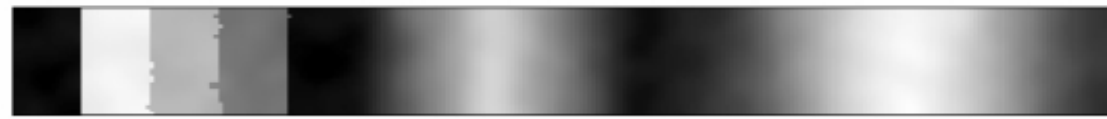

Fig. 4. (a) Synthetic real image. (b) Noisy data test image. Reconstructions computed with: (c) the weak membrane potential,(d) weak plate potential, (e) PARC-AL and (d) PARC-IL (see text).

(a)

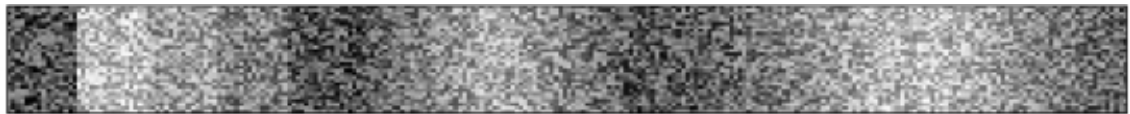

(b)

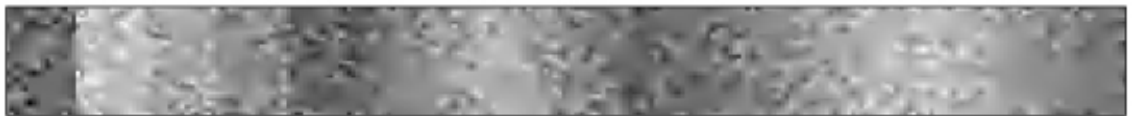

(c)

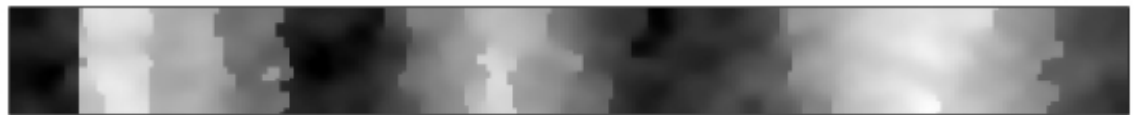

Fig. 5. (a) Noisy data test image. Reconstructions computed with: (b) weak plate potential and (c) PARC-AL

well-known effect is generated because the WTP changes the jumps by ramps. On the other hand, panels (e) and (f) show the reconstructions computed with the proposed PARC-AL and PARC-IL models, respectively. As one can note, the three different kinds of regions are reconstructed with high quality. The mean squared errors for the reconstructions are: $6.65 \mathrm{e}-4,5.04 \mathrm{e}-4$, and $4.09 \mathrm{e}-4$; for the panels: (d), (e) and (f), respectively. The data were normalized to the interval $[0,1]$ and corrupted with uniform noise with amplitude equal to 0.2, the MSE for the noisy data is $1.33 \mathrm{e}-2$. 
In our experiments, PARC models also have shown better performance for low signal to noise ratios. This is illustrated in figure 5. Panel (a) shows the corrupted image with uniform noise (with amplitude equal to 0.7 , that corresponds to a MSE equal to 0.162). Panels (b) and (c) show the restorations computed with the WTP and PARC-AL models, respectively. The MSE for the computed restorations were 0.022 ans 0.004 for WTP and PARC-AL models, respectively. In fact, as was noted by Geman and Reynolds [3], WTP model is unstable for low SNR.

\subsection{Comparison of PARC-AL vs. PARC-IL}

In order to compare the relative performance of the two presented PARC models (PARC-AL and PARC-IL), we performed the following experiment with a real test image. In Figure 6 panel (a) is shown the cameraman picture corrupted with gaussian noise with $\sigma^{2}=0.05$. Panel (b) and (c) the reconstructions computed with the $\mathrm{AL}$ and the IL model, respectively. The MSE were $1.5 \mathrm{e}-3$ and $0.9 \mathrm{e}-$ 3 Corresponding details are presented in panels (d), (e) and (f). As one can appreciate, the IL model performs a better restoration of the details. However, the computational time is larger for the IL model: 15 secs. while the restoration with the AL model took 3 secs. in a pentium III at $800 \mathrm{Mhz}$. based computer. The computational time for the WTP model (results not shown) was of 23 secs. We noted that for this case of high SNR, the restoration of the WTP model (with MSE equal to 1.6e-3) looks very similar to the one computed with the PARCAL model. It should be noted that the convergence of the ANLCG minimization algorithm, used in the PARC-IL case, can always be guaranteed, which is not the case for PARC-AL; we have tested this last algorithm extensively, however, and it never has failed to converge.

\subsection{Second Order Anisotropic Diffusion}

Refs. [18] 15] shown the relationship between robust potentials and anisotropic diffusion. Following that work, one can get second order anisotropic diffusions based on PARC potentials, for instance

$$
f^{t+1}=f^{t}+h \frac{\partial}{\partial f_{r}^{t}} \sum_{q, s \in N_{r}} \rho_{p a r c}(f)_{r},
$$

where $\rho_{\text {parc }}(f)_{r}$ is given in (15), $f^{0}$ corresponds to the image in 66 (a) and the step size $h$ is chosen small enough. Figure 7 shown images of a sequence computed with this method. We use the same PARC potential than in the experiment of figure 6 .

\section{Discussion and Conclusions}

We have presented a new model for edge-preserving regularization, that allows one to reconstruct second order piecewise smooth images. This model is based 


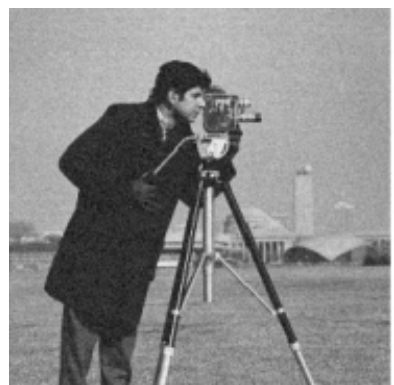

(a)

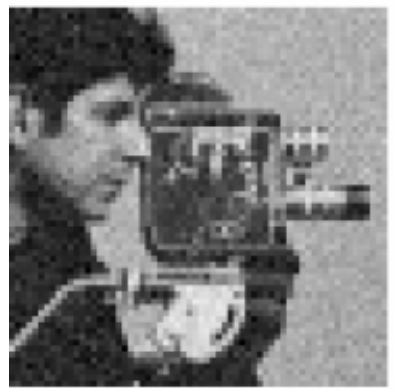

(d)

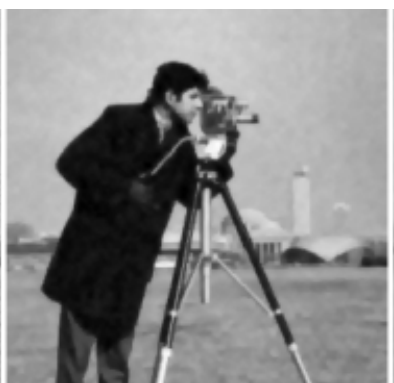

(b)

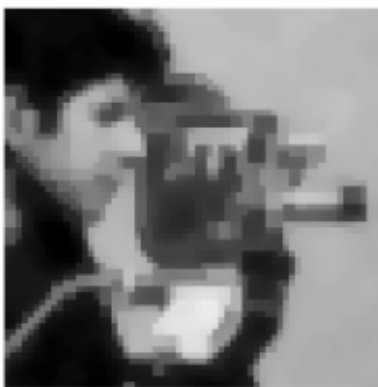

(e)

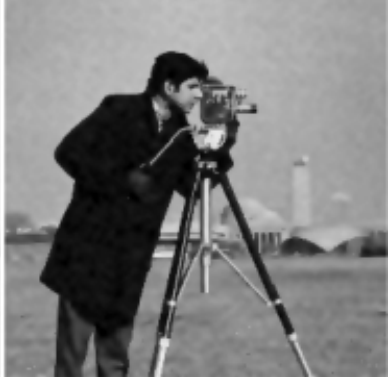

(c)

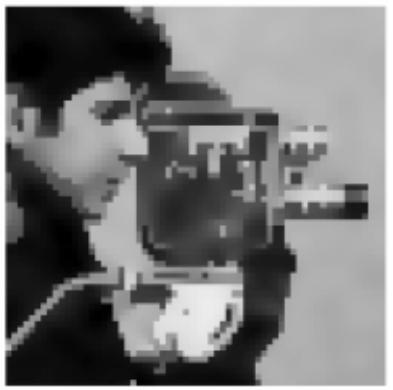

(f)

Fig. 6. (a) Cameraman picture corrupted with uniform noise. Reconstructions computed with: (b) PARC-AL and (c) PARC-IL. (d), (e), (f): corresponding details.
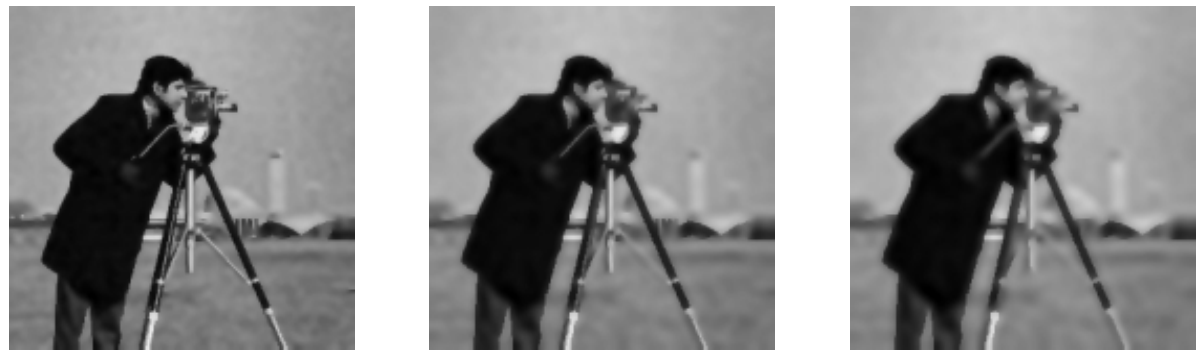

Fig. 7. Images of a sequence computed with an anisotropic diffusion algorithm based on PARC-IL potentials

on the physical analogy of adaptive rest condition potentials (ARC). In order to focus the paper on the characteristics of the ARC potentials, we dealt with the problem of restoring noisy images, but their use can be extended to other image processing and computer vision problems. We showed that the first order ARC model -which has been used before - is equivalent to the well known adaptive weak spring model, and hence, it has the problem of producing staircase-like solutions in regions of constant slope; to overcome this problem, we propose here 
an extension that generates new second order potentials; we called these potentials the thin plate model with adaptive rest condition (PARC). These PARC potentials have the property of changing their behavior to a first order EPR potential at the edges (steps in the gray level) of the image. As a result, regularized cost functionals based on the PARC model are more stable and perform a better restoration of edges and smooth regions, because PARC potentials extend the definition of smoothness to include regions with almost constant slope.

We introduced two kinds of PARC potentials: the PARC with analog line process (PARC-AL) and the PARC with implicit line process (PARC-IL). We found that these families have specific advantages:

$P A R C-A L$ potentials generate a pair of coupled systems (in general, linear for the restored image and non-linear for the auxiliary variables) that can be alternatively minimized. A special case results from selecting a coupled quadratic potential; in such case the resulting coupled systems are linear and can be efficiently minimized (in our case, we used the Gauss-Seidel algorithm in an alternated scheme). This model can incorporate potentials that penalize specific configurations of the auxiliary variable (for example the thickness of the edges; see [41415] for more details).

$P A R C-I L$ potentials are non-linear; in this case, the line process is implicitly represented by a detection function that depends on the image gradient; for minimizing the corresponding cost functionals, we proposed a modified Nonlinear Conjugate Gradient Algorithm, for which one can guarantee convergence (at least to a local minimum). Experiments have shown that (in general) one obtains better reconstructions with the PARC-IL model although at a higher computational cost. An interesting open theoretical problem is to determine the precise relation between PARC-AL and PARC-IL potentials, as has been found in the case of WSS models.

Acknowledgments. This work was funded in part by CONACYT, Mexico under grant 34575-A.

\section{References}

1. Li, S.Z.: Markov Random Field Modeling in Image Analysis, Springer-Verlag, New York (2001)

2. Geman, S., Geman, D.: Stochastic relaxation, Gibbs distributions and Bayesian restoration of images, IEEE Trans. Pattern Anal. Machine Intell., 6 (1984) 721741

3. Geman, D., Reynolds, G.: Constrained restoration and the recovery of discontinuities, IEEE Trans. Image Processing, 14 (1992) 367-383

4. Black, M.J., Rangarajan, A.: Unification of line process, outlier rejetion, and robust statistics with application in early vision, Int. Journal of Computer Vision, 19 (1996) 57-91

5. Charbonnier, P., Blanc-Féraud, L., Aubert, G., Barlaud, M.: Deterministic edgepreserving regularization in computer imagining, IEEE Trans. Image Processing, 6 (1997) 298-311 
6. Kubota, T., Huntsberg, T.: Adaptive anisotropic parameter estimation in the weak membrane model, Proc. Int WS in EMMCVPR'97, Lecture Notes in Computer Vision 1223, Springer Verlag, Venice Italy, (1997) 179-194

7. Geman, S., McClure, D.E.: Bayesian image analysis methods: An application to single photon emission tomography, in Proc. Statistical Computation Section, Amer. Statistical Assoc., Washington, DC, (1985) 12-18

8. Blake A., Zisserman, A.: Visual reconstruction, The MIT Press, Cambridge, Massachusetts (1987)

9. Geman, D., Yang, C.: Nonlinear image recovery with half-quadratic regularization, IEEE Trans. Image Processing, 4 (1995) 932-946

10. Cohen, L.: Auxilar varibales and two-steps iterative algorithms in computer vision problems, Journal Mathematical Imaging and Vision, 6 (1996) 59-83

11. Ambrosio, L., Tortorelli, V.M.: Approximation of functionals depending on jumps by elliptic functionals via $\Gamma$-convergence, Commun. Pure Appl. Math, 43 (1990) 999-1036

12. Rivera, M., Marroquin, J.L.: The adaptive rest condition spring model: an edge preserving regularization techinque, in Proc. IEEE-ICIP 2000, Vol. II, Vancouver, BC, Canada (2000) 805-807

13. Rivera, M., Marroquin, J.L.: Efficient Half-Quadratic Regularization with Granularity Control, Techical Report: 05.06.2001, I-01-07 (CC), CIMAT, Mexico (2001)

14. Teboul, S., Blanc-Féraud, L., Aubert, G., Barlaud,M., Variational approach for edge-preserving regularization using coupled PDE's, IEEE Trans. Image Processing, 7 (1998) 387-397

15. Black, M.J., Sapiro, G., Marimont D.H., Heeger, D.: Robust anisotropic diffusion, IEEE Trans. Image Processing, 7 (1998) 421-432

16. Idier, J.: Convex half-quadratic criteria and interactig auxiliar variables for image restoration, IEEE Trans. Image Processing, 10 (2001) 1001-1009

17. Nocedal J., Wright, S.J.: Numerical Optimization (Springer Series in Operations Research), Springer-Verlag, New York (1999)

18. Perona, P., Malik, J.: Scale-space and edge detection using anisotropic diffusion, IEEE Trans. Pattern Anal. Machine Intell., 12 (1990) 629-639 